\title{
A Physically-Based BSDF for Modeling the Appearance of Paper
}

\author{
Marios Papas, Krystle de Mesa, and Henrik Wann Jensen
}

University of California, San Diego

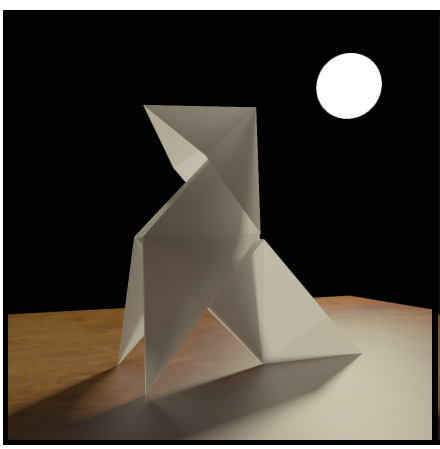

(a)

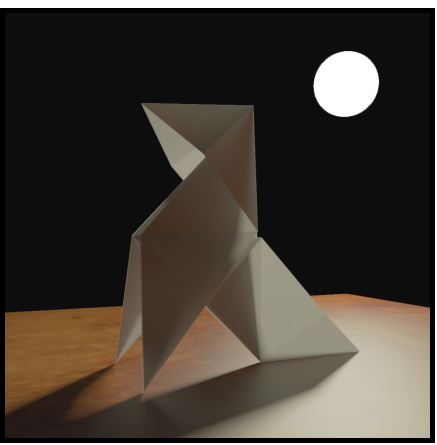

(b)

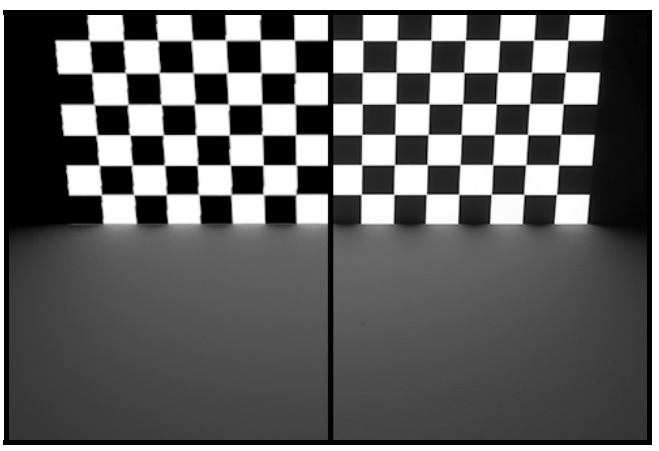

Paper BSDF

Photograph

Figure 1: A back-lit origami bird rendered with our Paper BSDF at varying paper thicknesses: (a) $0.05 \mathrm{~mm}$, (b) $0.26 \mathrm{~mm}$ (our measured parameter for matte paper). On the right, a comparison between a photograph (right side) and our Paper BSDF for matte paper (left side). The scene is of an illuminated checkerboard pattern with matte paper placed in front of it.

\begin{abstract}
We present a novel appearance model for paper. Based on our appearance measurements for matte and glossy paper, we find that paper exhibits a combination of subsurface scattering, specular reflection, retroreflection, and surface sheen. Classic microfacet and simple diffuse reflection models cannot simulate the double-sided appearance of a thin layer. Our novel BSDF model matches our measurements for paper and accounts for both reflection and transmission properties. At the core of the BSDF model is a method for converting a multi-layer subsurface scattering model (BSSRDF) into a BSDF, which allows us to retain physically-based absorption and scattering parameters obtained from the measurements. We also introduce a method for computing the amount of light available for subsurface scattering due to transmission through a rough dielectric surface. Our final model accounts for multiple scattering, single scattering, and surface reflection and is capable of rendering paper with varying levels of roughness and glossiness on both sides.
\end{abstract}

Categories and Subject Descriptors (according to ACM CCS): I.3.7 [Computer Graphics]: Three-Dimensional Graphics and Realism - Color, shading, shadowing, and texture

\section{Introduction}

Although paper is a material used in our everyday lives, its complex structure is often overlooked. In detail, paper is composed of pulp fibers ranging from wood and cotton to papyrus and silk, all of which is mixed with water and results in unique microstructures for the paper's surface (Figure 3). Additionally, most paper manufacturers typically include different layers of material to enhance the paper quality. For today's typical ink jet coated papers, this often includes an ink absorbing layer, which may consist of fine silica and water soluble binders, as well as reflective and back coating layers. 


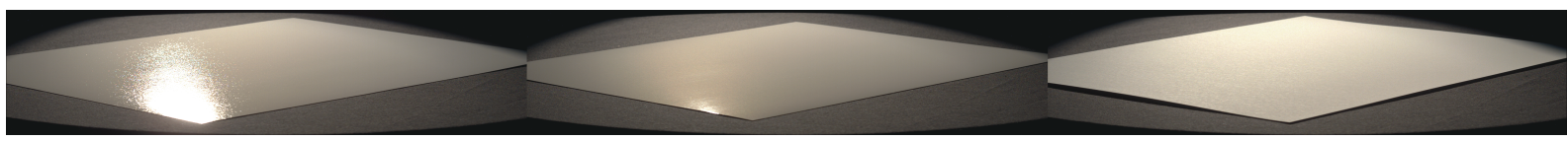

Figure 2: Papers used to evaluate our model (from left to right): luster photo paper, glossy photo paper, and matte presentation paper. All images were subjected to the same illumination conditions.

Paper can also be treated with fluorescent brightening agents for whitening and polished to increase the glossiness. Figure 2 exemplifies how these different types of manufacturing processes contribute to the overall appearance of paper.

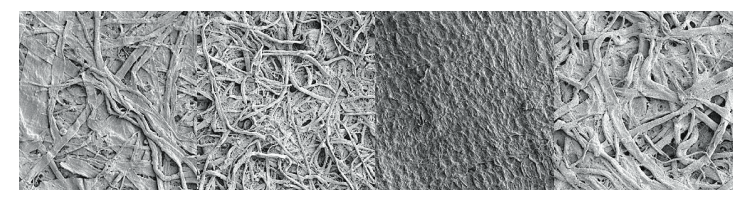

Figure 3: Paper at 100X magnification (from left to right): wood, silk, papyrus, and cotton paper (Images courtesy of The Paper Project http: //www. paperproject.org/).

The detailed composition of paper has not been fully investigated within appearance modeling research. We have performed thorough reflection and transmission measurements of light scattering from different types of paper and found that paper is a highly scattering, optically thick material, which exhibits a combination of subsurface scattering, specular reflection, retroreflection, surface sheen, and transmission. Existing appearance models do not account for these phenomena. Microfacet based BSDF models have simple parameters to fit and they have been used for modeling specular reflection and surface sheen, but they do not account for subsurface scattering. The various BSSRDFs based on light diffusion theory could be used to fit parameters on transmission and reflection through thin, layered slabs [DJ05, DI11, HCJ13]; however, in the case of thin sheets of paper, we uniformly illuminate all surface regions that contribute to the measurement location, thus allowing us to reduce the BSSRDF into a BSDF.

We introduce a novel BSDF for paper that simulates the scattering behavior observed in our measurements and can be used to recover physically meaningful parameters. We model paper as a thin, highly scattering homogeneous medium coated with two (possibly different) rough dielectric boundaries. Our model uses a BSDF reduction of the multi-layer model [DJ05], single scattering theory [HK93], and a microfacet model for surface reflection and refraction [WMLT07]. Furthermore, we propose a method for evaluating light attenuation through rough surfaces using the BTDF of both the front and back surfaces of paper.

We verify the accuracy of our BSDF model with a nonlinear constrained minimization algorithm that allows us to generate parameters for our paper measurements such that the rendered results closely match actual measured BSDFs. Results show that our model is able to match our measurements and it can be used to reproduce the appearance of both matte and glossy types of paper.

\section{Related Work}

Paper has been previously studied by Bartman et al. [BCS64], who reveal that paper consists of a non-Lambertian surface. Chen [Che09] investigates the accuracy of scattering approximations for paper using collimated light, and Green et al. [GLL00] present a stochastic model for simulating the fiber structure of paper. Fabritius et al. [FSM06] studies the refractive index of paper.

Microfacet models were first introduced by Torrance and Sparrow [TS67] to describe the surface reflection from rough, metallic surfaces. Each surface is modeled as a collection of symmetric V-cavities through a normalized microfacet distribution function and uses a geometric term to capture masking and shadowing effects from each facet. Microfacet theory was later applied in computer graphics [Bli77, CT82] and extended [War92] to include anisotropic materials. For rough, diffuse materials, Oren and Nayar [ON94] introduced a model that describes each microfacet as Lambertian, and van Ginneken et al. [vGSK98] use microfacets to account for diffuse and specular reflectance from rough surfaces. Weidlich and Wilkie [WW07] layer several microfacet surfaces in order to describe a single BRDF model for smooth and rough multi-layer surfaces. Dai et al. [DWL ${ }^{*} 09$ ] introduced a dual-microfacet model that approximates light transmission through two surfaces of different roughness with a spatiallyvarying distribution function of the microfacet's normal.

Walter et al. [WMLT07] present a microfacet BSDF for rough glass. In our method, we use the rough glass BSDF to model surface reflection and refraction at the layer boundaries. Along with their BSDF model, they present the GGx microfacet normal distribution function. We found that this distribution works extremely well in cases with high roughness like matte paper.

Stam [Sta01] presented a model for a skin layer, which accounts for light scattering through a thin layer bounded by rough dielectric BSDFs. While accurate and general, this model does not scale to smooth, almost specular, dielectric surfaces such as glossy paper. The lack of scalability is due to two aspects of this technique: Stam projects the boundary $\mathrm{BSDF}$ into a frequency space representation by numerically 
integrating it against Fourier basis functions, which does not work well for high frequency reflectance functions. Secondly, the final BSDF is computed by solving a system of ordinary differential equations using a matrix exponential. In the presence of peaked reflection and transmission functions, the system becomes very large, leading to impracticably slow linear algebra computations.

Mahan [Mah95] provides an analytic solution to the Boltzmann's equation to model diffusion of non-absorbing particles through a thick slab. In this work, absorption within the slab is assumed to be zero, and surface effects, such as roughness and index of refraction differences at the slab boundaries, are not handled.

A BSSRDF model was first presented in computer graphics by Jensen et al. [JMLH01]. This BSSRDF uses a dipole diffusion approximation to simulate light scattering in homogeneous, semi-infinite, translucent materials. Donner and Jensen [DJ05] extend the BSSRDF with the multipole to account for the diffusion of light in thin slabs and multi-layer materials.

Other BSSRDF-based capture methods [GLL*04, TWL*05, PvBM*06, WZT*08] measure diffusion profiles to approximate their model's parameters. These methods rely on measuring scattering profiles with large extent, which is something not present in paper. Additionally, these methods assume that reflectance is mainly due to multiple scattering, thus ignoring directional effects such as single scattering and surface reflection. Ghosh et al. [GHP*08] present a method for modeling and measuring layered facial reflectance from 20 photographs and account for surface reflectance, single scattering and multiple scattering.

A time-resolved analytic diffusion model was presented by Patterson et al. [PCW89] for measuring optical properties of tissue. However, the model does not account for rough surfaces. They provide spatially and time resolved solutions to diffusion equations and also provide an analytic time-resolved BRDF for reflectance. They note that this time-resolved reflectance BRDF can improve the signal to noise ratio in their measurements. An analytic BRDF was also derived for the Jensen et al. BSSRDF [JMLH01] which was used as part of their parameter fitting process.

In our method, we chose to use the multi-layer BSSRDF [DJ05] as a starting point for modeling multiple scattering in paper. In the special case of thin, highly scattering, and optically thick slabs like paper, we show that the BSSRDF can be reduced to an analytic BSDF. We modify the multi-layer model to better account for the possible rough boundaries of paper by using the BSDF of Walter et al. [WMLT07]. For modeling single scattering, we use the Hanrahan and Krueger BSDF [HK93]. Although the multi-layer model [DJ05] has never been verified through measured data, our BSDF measurements validate its accuracy in the case of a single, highly scattering and optically thick layer.

\section{BSDF Measurements of Paper}

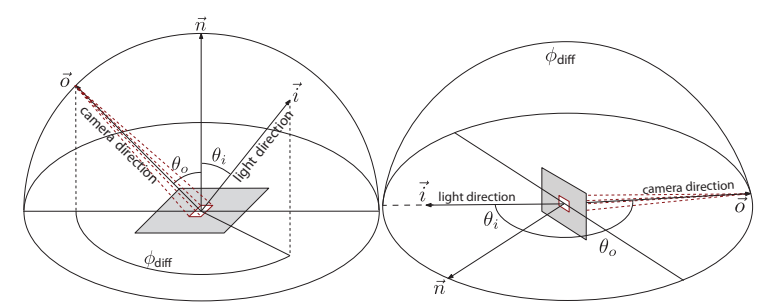

Figure 4: Our BRDF and BTDF acquisition setup.

We measured several types of commercially available paper and found that they can be well-modeled by an isotropic BSDF. Additionally, we found that the appearance characteristics can be represented by a set of the following paper types: Epson Ultra Premium Presentation Paper Matte, Epson Ultra Premium Photo Paper Luster (a rough, glossy type of paper), and Epson Ultra Premium Photo Paper Glossy. We focus on these papers not only because they contain a considerable amount of variation from one another, but also because of their representation as the standard types of paper widelyused today. Figure 2 highlights the visual difference of each type of paper.

Figure 4 illustrates our acquisition setup. For each paper sample, we performed full hemispherical BRDF measurements in 10 degree increments as well as in-plane BTDF measurements every 5 degrees. The paper samples were illuminated on its front surface with a fiber optic light guide attached to a 150-watt quartz halogen bulb with DC-regulated output. We sampled both the front surface for its BRDF and the back surface for its BTDF. HDR images were acquired with a 1600x1200 resolution CCD camera, and for each shutter speed stop, 5 images were averaged to remove random noise. The resulting image was then subtracted against a black image that visualized fixed pattern noise for that shutter speed. We only processed a small rectangular region in the center portion of each paper with a fixed width of 256 pixels and a variable height relative to the cosine of the angle between the surface normal and camera direction, $\theta_{0}$. This accommodated the decreased sampling size of the paper as the camera approaches grazing angles. For our BSDF acquisition, we follow the parametrization of Matusik et al. [MPBM03], which proposes reducing the BRDF sample ranges for isotropic materials. This method allows us to sample $\theta_{i}$ from $0^{\circ}$ to $90^{\circ}, \theta_{o}$ from $0^{\circ}$ to $90^{\circ}$, and $\phi_{\text {diff }}$ from $0^{\circ}$ to $180^{\circ}$, thus reducing the lengthy acquisition procedure associated with these types of intricate measurements. For each measurement, the acquisition time amounted to approximately two days.

\subsection{Reflection}

Matte Paper Our BRDF measurements for matte paper indicate a relatively high amount of roughness that is characterized by varying levels of grazing angle sheen and retroreflection, as well as the lack of peaks in reflectance near the 

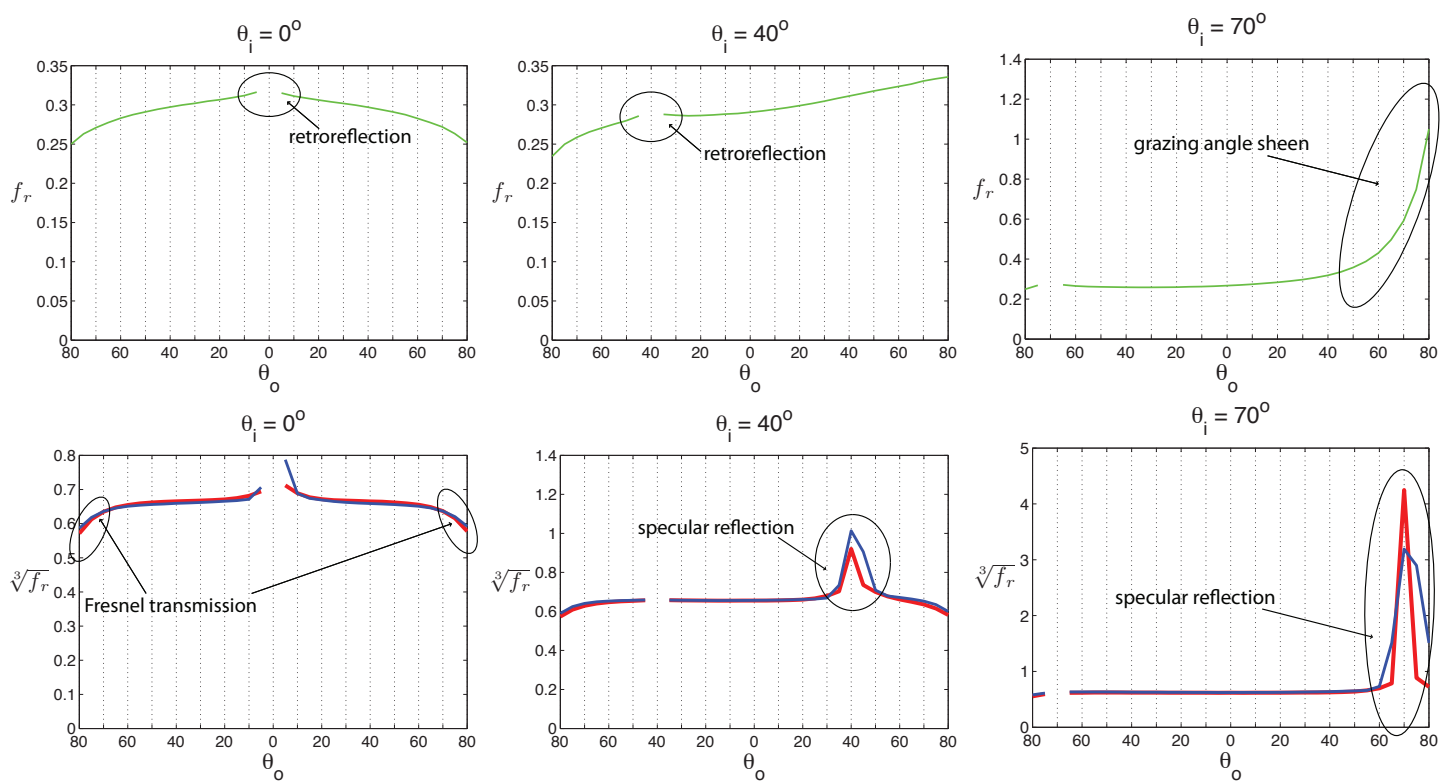

Figure 5: In-plane slice $\left(\phi_{\text {diff }}=180^{\circ}\right)$ of our full BRDF measurements for matte (green) paper (top), with glossy (red) and luster (blue) photo paper (bottom). Gaps in the measurements result from when the light blocks the camera.
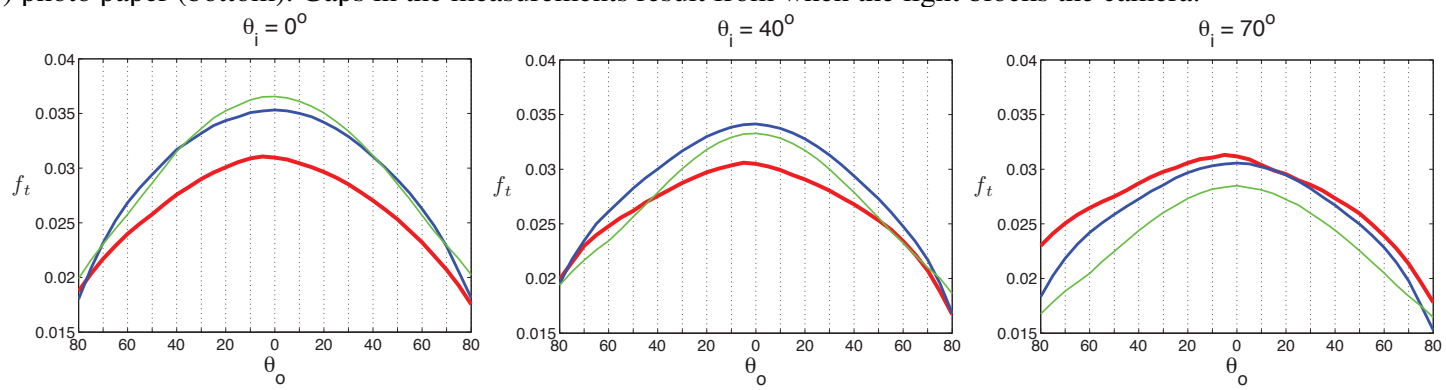

Figure 6: In-plane $\left(\phi_{\text {diff }}=180^{\circ}\right)$ BTDF measurements for matte (green), glossy (red), and luster (blue) paper. Camera angles, $\theta_{o}$, are with respect to $-\vec{n}$.

specular direction. We describe grazing angle sheen as significant increases in reflectance when both $\theta_{i}$ and $\theta_{o}$ are at grazing angles, and it can be attributed to rough surface reflection and single scattering within the material. Retroreflection occurs when the material reflects light back towards the light source; this is highlighted in Figure 5 with the slight peak as $\theta_{o}$ approaches $\theta_{i}$.

The wide, smooth bump in Figure 5 when $\theta_{i}=0$ is produced by multiple scattered light that becomes attenuated by the rough surface. This contrasts with smooth surfaces where the light attenuation is fairly constant at angles with less than $60^{\circ}$ deviation from the surface normal.

Luster and Glossy Photo Paper Both luster and glossy photo paper have a smooth front surface due to their glossy coating, which produces varying levels of specular reflection rather than retroreflection (Figure 5). However, luster paper's front surface is more rough than glossy paper, as hinted by its overall wider specular peak.
Luster and glossy paper's multiple scattered light attenuation is relatively constant except when $\theta_{o}$ approaches grazing angles (see Figure 5). This characteristic is also present in the Fresnel equations. Specifically, Fresnel reflection rapidly increases at these angles, while transmission decreases with the same trend shown in the glossy measurements.

\subsection{Transmission}

The BTDF measurements for matte, luster, and glossy paper are similar and approximately vary by a scaling factor for each material (Figure 6). All three measured BTDFs lack directionally dependent behavior, with respect to the incident direction $\theta_{i}$, without showing any peaks in transmittance near the expected transmission direction. This indicates that our paper samples are highly scattering and optically thick. As a result, all directionality information of the light is lost as it travels through the medium and exits the back surface. With this information and an additional analysis of cross-polarized 


$$
\begin{array}{r}
f_{r}^{(s, 1, m)}(\vec{i}, \vec{o})= \begin{cases}f_{r}^{(s)}\left(\vec{i}, \vec{o}, \eta_{f}, m_{f}\right)+f_{r}^{(1)}\left(\vec{i}, \vec{o}, \eta_{f}, m_{f}\right)+f_{r}^{(m)}\left(\vec{i}, \vec{o}, \eta_{f}, m_{f}\right) & \text { if }(\vec{n} \cdot \vec{i})>0 \text { and }(\vec{n} \cdot \vec{o})>0, \\
f_{r}^{(s)}\left(\vec{i}, \vec{o}, \eta_{b}, m_{b}\right)+f_{r}^{(1)}\left(\vec{i}, \vec{o}, \eta_{b}, m_{b}\right)+f_{r}^{(m)}\left(\vec{i}, \vec{o}, \eta_{b}, m_{b}\right) & \text { if }(\vec{n} \cdot \vec{i})<0 \text { and }(\vec{n} \cdot \vec{o})<0, \\
0 & \text { otherwise }\end{cases} \\
f_{t}^{(1, m)}(\vec{i}, \vec{o})= \begin{cases}f_{t}^{(1)}\left(\vec{i}, \vec{o}, \eta_{f}, m_{f}, \eta_{b}, m_{b}\right)+f_{t}^{(m)}\left(\vec{i}, \vec{o}, \eta_{f}, m_{f}, \eta_{b}, m_{b}\right) & \text { if }(\vec{n} \cdot \vec{i})>0 \text { and }(\vec{n} \cdot \vec{o})<0, \\
f_{t}^{(1)}\left(\vec{i}, \vec{o}, \eta_{b}, m_{b}, \eta_{f}, m_{f}\right)+f_{t}^{(m)}\left(\vec{i}, \vec{o}, \eta_{b}, m_{b}, \eta_{f}, m_{f}\right) & \text { if }(\vec{n} \cdot \vec{i})<0 \text { and }(\vec{n} \cdot \vec{o})>0, \\
0 & \text { otherwise }\end{cases}
\end{array}
$$

Figure 7: The Paper BSDF.

measurements, we found that more than $99 \%$ of the transmitted light is due to multiple scattering. Additionally, all transmission measurements in Figure 6 showcase a prominent wide, smooth bump that is similar to the same feature mentioned in the matte paper BRDF measurements (Section 4). This is consistent with our observation that multiple scattered light attenuated through a rough surface exhibits this angular distribution.

As $\theta_{i}$ increases, the transmission values for each paper decrease, thus showing how light attenuates through the front surface differently. Since matte paper has a rough surface, less light is transmitted through the back surface (due to masking and shadowing) as $\theta_{i}$ deviates from the surface normal. In contrast, luster and glossy paper have smoother surfaces, which cause a slower rate of decrease at non-grazing angles and a more abrupt decrease near grazing angles.

\section{The Paper BSDF}

\begin{tabular}{cl}
\hline Symbol & Description \\
\hline$\vec{i}$ & Normalized incident light direction \\
$\vec{o}$ & Normalized scattered light direction \\
$\vec{n}$ & Normalized macro surface normal \\
\hline$\eta_{f}, \eta_{b}$ & Refraction index of front/back surface \\
$m_{f}, m_{b}$ & Front/back surface roughness \\
\hline$\sigma_{s}, \sigma_{a}$ & Scattering and absorption coefficients (in $\left.\mathrm{mm}^{-1}\right)$ \\
$g_{f}, g_{b}$ & Forward and back scattering mean cosine angles \\
$w_{f}, w_{b}$ & Forward and back scattering ratio \\
$d$ & Layer thickness (in $m m$ ) \\
\hline
\end{tabular}

Table 1: Paper BSDF Nomenclature

For our model we use a combination of physically-based BRDF and BTDF models. The resulting BSDF can be used to render paper-like materials with different sides varying in roughness and levels of gloss.

For rendering, we assume that paper is an optically thick and highly scattering homogeneous medium enclosed by two possibly different dielectric front and back surfaces. We further assume that the length of the mean free path is orders of magnitude smaller than the thickness of paper and the spatial resolution of the sensor. These assumptions enable the use of a BSDF, instead of a BSSRDF, for accurately modeling the appearance of paper.

In our model, we distinguish the front and back surface by using the macro surface normal, $\vec{n}$, which we define to always be located on the same side as the front surface. Table 1 provides an index of other main terms we use in our equations.

The Paper BSDF consists of a reflection and a transmission component. Reflection is further expanded to the following two terms: surface reflection, $f_{r}^{(s)}$, and reflection due to subsurface scattering, $f_{r}^{(1, m)}$, where $(1, m)$ denotes both single and multiple scattering, respectively. Transmission is divided into $f_{t}^{(1)}$ for single scattering and $f_{t}^{(m)}$ for multiple scattering. We also account for different refraction indices and roughness levels for both the front and back sides of paper. Using the sign of the dot products $(\vec{n} \cdot \vec{i})$ and $(\vec{n} \cdot \vec{o})$ allows us to determine which set of refraction and roughness parameters to use, as well as whether light is reflecting or transmitting through the material at a given point. Figure 7 summarizes the Paper BSDF final equation.

\subsection{Surface Reflection}

For surface reflection, $f_{r}^{(s)}(\vec{i}, \vec{o}, \eta, m)$, we use the BRDF component of the rough glass model introduced by Walter et al. [WMLT07]:

$$
f_{r}^{(s)}(\vec{i}, \vec{o}, \eta, m)=\frac{D\left(\vec{h}_{r}, m\right) F_{r}\left(\eta,\left(\vec{o} \cdot \overrightarrow{h_{r}}\right)\right) G\left(\vec{i}, \vec{o}, \vec{h}_{r}, m\right)}{4(\vec{n} \cdot \vec{i})(\vec{n} \cdot \vec{o})},
$$

with $D\left(\vec{h}_{r}, m\right)$ representing the distribution of the surface microfacets, $F_{r}\left(\eta,\left(\vec{o} \cdot \overrightarrow{h_{r}}\right)\right)$ representing the Fresnel reflection of the microfacets, and $G\left(\vec{i}, \vec{o}, \vec{h}_{r}, m\right)$ corresponding to the geometric attenuation term. For the microfacet distribution and geometric terms, we found that the GGx distribution provides a good estimation for rough surfaces such as matte paper, while the Beckmann distribution works best for smooth surfaces such as the front sides of luster and glossy paper.

\subsection{Surface Transmission}

Although we do not define a surface transmission term, $f_{t}^{(s)}$, in our final equation (Figure 7), surface transmission is negligible for the optical thickness of paper types we consider. 
Therefore, we use it only when accounting for transmitted light that enters and exits the front and back surface. Transmission through a dielectric boundary, $f_{t}^{(s)}$, is used for our subsurface scattering equations (discussed in subsequent sections). Walter et al. [WMLT07] define $f_{t}^{(s)}\left(\vec{i}, \vec{o}, \eta_{i}, \eta_{o}, m\right)$ as:

$$
f_{t}^{(s)}=\frac{\left|\left(\vec{i} \cdot \vec{h}_{t}\right)\left(\vec{o} \cdot \vec{h}_{t}\right)\right|}{|(\vec{n} \cdot \vec{i})(\vec{n} \cdot \vec{o})|} \frac{\eta_{o}^{2} D\left(\vec{h}_{t}, m\right) F_{t}\left(\frac{\eta_{o}}{\eta_{i}},\left(\vec{o} \cdot \vec{h}_{t}\right)\right) G\left(\vec{i}, \vec{o}, \vec{h}_{t}, m\right)}{\left(\eta_{i}\left(\vec{i} \cdot \overrightarrow{h_{t}}\right)+\eta_{o}\left(\vec{i} \cdot \vec{h}_{t}\right)\right)^{2}}
$$

where $\vec{h}_{t}=\frac{-\left(\eta_{i} \vec{i}+\eta_{o} \vec{o}\right)}{\left\|\left(\eta_{i} \vec{i}+\eta_{o} \vec{o}\right)\right\|^{2}}$ is the normalized transmission half angle vector.

\subsection{Light Attenuation from Smooth or Rough Surfaces}

In the case of smooth dielectric surfaces such as the coating of glossy and luster paper, the Fresnel transmission equations provide a good match against our measurements. However, we found that the Fresnel transmission equation is not a good choice for rough surfaces like matte paper. In previous work [DJ05, GHP*08, DI11, HCJ13], light attenuation from rough surfaces is estimated by integrating the surface BRDF and assuming that non-reflected light is transmitted. This approximation performs poorly when compared against our measurements; as a result, we directly evaluate the amount of light that transmits through a rough surface by numerically integrating the surface BTDF (Equation 4). Our equation that approximates the light attenuation through both smooth and rough surfaces is:

$$
\operatorname{Att}(\vec{i}, \eta, m)= \begin{cases}1-F_{r}(\eta,(\vec{n} \cdot \vec{i})) & \text { if } m<t \\ \rho_{d t}(\vec{i}, \eta, m) & \text { otherwise }\end{cases}
$$

where $t$ is a threshold that defines a level of smoothness on the surface such that shadowing and masking is insignificant. As roughness level $m$ decreases, the number of samples required for the numerical integration $\rho_{d t}$ increases, making this evaluation very costly. However, Figure 8 shows that $\rho_{d t}$ with the Beckmann distribution closely matches the Fresnel transmission equation when $m$ is 0.05 . This indicates that we can avoid the expensive numerical integration in these cases by using the analytic Fresnel equations. We chose $t=0.05$ as a lower bound when using the Beckmann distribution, in order to indicate when the surface roughness should be numerically integrated. We define $\rho_{d t}$ as

$$
\begin{aligned}
& \rho_{d t}(\vec{i}, \eta, m)= \\
& \quad=\int_{\vec{o} \in \Omega_{-(\vec{n})}} f_{t}^{(s)}(\vec{i}, \vec{o}, \eta, 1, m)|\vec{n} \cdot \vec{o}| d \vec{o} \\
& \quad \approx \frac{\pi^{2}}{N} \sum_{\phi_{o}=0}^{2 \pi} \sum_{\theta_{o}=\pi / 2}^{\pi} f_{t}^{(s)}(\vec{i}, \vec{o}, \eta, 1, m)\left|\cos \theta_{o}\right| \sin \theta_{o},
\end{aligned}
$$

where $f_{t}^{(s)}$ uses the same distribution as the corresponding $f_{r}^{(s)}$ (GGx or Beckmann). Rather than performing the numerical integration for every incoming direction, index of refraction, and roughness, we instead evaluate the smooth $\rho_{d t}$ function at various points along its $3 \mathrm{D}$ domain and then use interpolation [dL07] during both fitting and rendering. This considerably improves the evaluation time needed for calculating the surface attenuation.

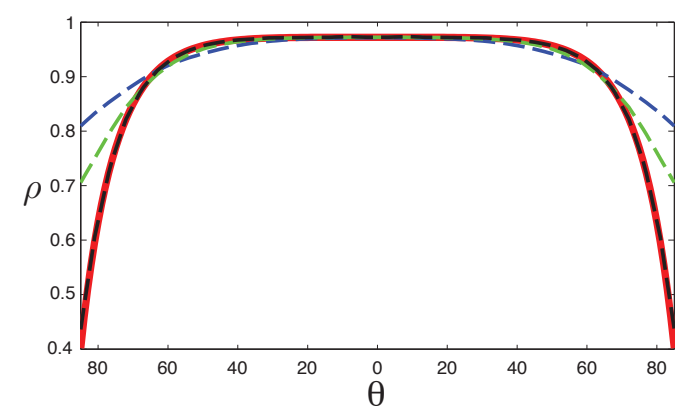

Figure 8: A comparison plot of $\rho_{d t}$ with 0.45 (blue), 0.25 (green), and 0.05 (black) roughness values with the Fresnel transmission equation (red) when $\eta_{o}=1.4$. When roughness is equal to $0.05, \rho_{d t}$ becomes nearly identical to the Fresnel transmission equation. As the roughness increases, $\rho_{d t}$ approaches the wide, smooth bump shape seen in our rough BTDF measurements (Figure 6).

\subsection{Single Scattered Reflection}

We account for single scattered reflection by applying our surface attenuation term (Equation 5) to the single scattering model [HK93], $f_{r}^{(1)}(\vec{i}, \vec{o}, \eta, m)$, as follows:

$$
f_{r}^{(1)}=\operatorname{Att}(\vec{i}, \eta, m) \operatorname{Att}(\vec{o}, \eta, m) \frac{\alpha p(-\vec{i}, \vec{o})\left(1-e^{\frac{-\tau_{d}}{|\vec{n} \cdot \vec{i}|}+\frac{-\tau_{d}}{|\vec{n} \cdot \vec{o}|}}\right)}{|\vec{n} \cdot \vec{i}|+|\vec{n} \cdot \vec{o}|},
$$

where $\alpha=\frac{\sigma_{s}}{\sigma_{a}+\sigma_{s}}$ is the albedo, $\sigma_{s}$ is the scattering coefficient, $\sigma_{a}$ is the absorption coefficient, $\operatorname{Att}(\vec{i}, \eta, m)$ is the fraction of light transmitted into the surface, and $\operatorname{Att}(\vec{o}, \eta, m)$ is the fraction of light that is transmitted outwards. Based on the assumption that the measured material is homogeneous, the optical depth is defined as $\tau_{d}=\sigma_{t} d$, where $\sigma_{t}=\sigma_{a}+\sigma_{s}$ is the extinction coefficient and $d$ is the thickness of the material.

We model retroreflection by applying a backscattering component to the Henyey-Greenstein phase function, $p(-\vec{i}, \vec{o})$ The Henyey-Greenstein phase function accounts for both backscattered and forward-scattered light from a multi-lobed phase function and is given by:

$$
p(-\vec{i}, \vec{o})=w_{b} p_{H G}\left(-\vec{i}, \vec{o}, g_{b}\right)+w_{f} p_{H G}\left(-\vec{i}, \vec{o}, g_{f}\right),
$$

where $g_{b}$ and $w_{b}$ is the mean cosine angle and weight for backscattering, $g_{f}$ and $w_{f}$ is the mean cosine angle and a weight for forward scattering, $w_{b}+w_{f}=1$, and

$$
p_{H G}(-\vec{i}, \vec{o}, g)=\frac{1}{4 \pi} \frac{1-g^{2}}{\left(1+g^{2}-2 g(\vec{i} \cdot \vec{o})\right)^{3 / 2}} .
$$




\subsection{Single Scattered Transmission}

We use the transmission component of the Hanrahan and Krueger model [HK93] to compute the amount of transmitted single scattered light that travels through the medium, $f_{t}^{(1)}\left(\vec{i}, \vec{o}, \eta_{i}, m_{i}, \eta_{o}, m_{o}\right)$.

$$
\text { If }(\vec{n} \cdot \vec{i})+(\vec{n} \cdot \vec{o}) \neq 0 \text {, then } f_{t}^{(1)} \text { is defined as: }
$$$$
f_{t}^{(1)}=\operatorname{Att}\left(\vec{i}, \eta_{i}, m_{i}\right) \operatorname{Att}\left(\vec{o}, \eta_{o}, m_{o}\right) \frac{\alpha p(-\vec{i}, \vec{o})\left(e^{\frac{-\tau_{d}}{|\vec{n} \cdot \vec{i}|}}-e^{\frac{-\tau_{d}}{|\vec{n} \cdot \vec{\partial}|}}\right)}{|\vec{n} \cdot \vec{i}|-|\vec{n} \cdot \vec{o}|} .
$$

Otherwise, if $(\vec{n} \cdot \vec{i})+(\vec{n} \cdot \vec{o})=0$, then $f_{t}^{(1)}$ is given by:

$$
f_{t}^{(1)}=\operatorname{Att}\left(\vec{i}, \eta_{i}, m_{i}\right) \operatorname{Att}\left(\vec{o}, \eta_{o}, m_{o}\right) \frac{\alpha \tau_{d} p(-\vec{i}, \vec{o}) e^{\frac{-\tau_{d}}{|\vec{n} \cdot \vec{b}|}}}{|(\vec{n} \cdot \vec{i})(\vec{n} \cdot \vec{o})|} .
$$

\subsection{Multiple Scattered Reflection}

For multiple scattered reflection, $f_{r}^{(m)}$, we reduce a single layer of the multi-layer BSSRDF [DJ05] to a BSDF. When a slab is uniformly lit and both viewed and illuminated from the same side, reflection due to multiple scattering can be evaluated by:

$$
f_{r}^{(m)}(\vec{i}, \vec{o}, \eta, m)=\operatorname{Att}(\vec{i}, \eta, m) \frac{R_{d}}{\pi} \operatorname{Att}(\vec{o}, \eta, m),
$$

with the reflection term, $R_{d}$, defined as:

$$
R_{d}=2 \pi \int_{0}^{\infty} R(r) r d r .
$$

The term $R_{d}$ accounts for all multiple scattered light in this configuration. Since in our measurements, incident illumination is uniform, incident radiance is not dependent on $r$ and the integral of $R(r)$ (Equation 14) can be reduced to an analytic formula:

$$
\begin{aligned}
R_{d} & =2 \pi \int_{0}^{\infty} R(r) r d r \\
& =\frac{\alpha^{\prime}}{2} \sum_{i=-n}^{n}\left(\operatorname{sign}\left(z_{r, i}\right) e^{-\sigma_{t r}||_{r, i} \mid}-\operatorname{sign}\left(z_{v, i}\right) e^{-\sigma_{t r}\left|z_{v, i}\right|}\right) .
\end{aligned}
$$

where $n$ is the number of dipoles used. The detailed derivation of $R_{d}$ can be found in our supplemental materials.

\subsection{Multiple Scattered Transmission}

Similar to Section 4.6, we define the BTDF for multiple scattering:

$$
f_{t}^{(m)}\left(\vec{i}, \vec{o}, \eta_{i}, m_{i}, \eta_{o}, m_{o}\right)=\operatorname{Att}\left(\vec{i}, \eta_{i}, m_{i}\right) \frac{T_{d}}{\pi} \operatorname{Att}\left(\vec{o}, \eta_{o}, m_{o}\right),
$$

with the transmission term, $T_{d}$, defined as:

$$
T_{d}=2 \pi \int_{0}^{\infty} T(r) r d r
$$

Assuming that the back surface is uniformly illuminated, the amount of light that arrives at the other surface of the material due to multiple scattering is:

$$
\begin{aligned}
T_{d} & =2 \pi \int_{0}^{\infty} T(r) r d r \\
& =\frac{\alpha^{\prime}}{2} \sum_{i=-n}^{n}\left(\operatorname{sign}\left(d-z_{r, i}\right) e^{-\sigma_{t r}\left|d-z_{r, i}\right|}-\right. \\
& \left.\quad \operatorname{sign}\left(d-z_{v, i}\right) e^{-\sigma_{t r}\left|d-z_{v, i}\right|}\right) .
\end{aligned}
$$

The detailed derivation of $T_{d}$ can be found in our supplemental materials.

\subsection{Model Parameters}

In its most general instantiation, our model is controlled by a set of 10 physically meaningful parameters. The parameters for the front and back boundary surfaces are: relative index of refraction $\left(\eta_{f}, \eta_{b}\right)$ and surface roughness $\left(m_{f}, m_{b}\right)$. The medium parameters are the scattering $\left(\sigma_{s}\right)$ and absorption $\left(\sigma_{a}\right)$ coefficients (given in $\mathrm{mm}^{-1}$ ), along with the layer thickness in $\mathrm{mm}(d)$. The multi-lobed phase function is parametrized by the forward $\left(g_{f}\right)$ and back $\left(g_{b}\right)$ scattering mean cosine angles along with the ratio of forward scattering events $\left(w_{f}\right)$. The ratio of the back scattering events $w_{b}=1-w_{f}$ is dependent on $w_{f}$. All parameters of the Paper BSDF are summarized in Table 1.

\section{Fitting}

The goal of the fitting algorithm is to find an optimal set of parameters $\vec{P}_{\text {opt }}$ for the BSDF that matches each of our measurements as closely as possible. We define $\vec{P}_{\text {opt }}$ as a set of parameters within a valid and physically meaningful range $\vec{P}_{\text {valid }}$, such that the difference between the BSDF model and our measurements, $M$, is minimized. The optimization problem is defined as follows:

$$
\begin{array}{r}
\underset{\vec{P} \in \vec{P}_{\text {valid }}}{\arg \min _{(\vec{i}, \vec{o}) \in M_{r}}} \sum_{(\vec{i}, \vec{o}) \in M_{t}}\left(\frac{\sqrt[3]{f_{r}^{(s, 1, m)}(\vec{i}, \vec{o}, \vec{P})}-\sqrt[3]{M_{r}(\vec{i}, \vec{o})}}{\sqrt[3]{\kappa\left(M_{r}\right)}}\right)^{2}+ \\
\sum\left(\frac{\sqrt[3]{f_{t}^{(1, m)}(\vec{i}, \vec{o}, \vec{P})}-\sqrt[3]{M_{t}(\vec{i}, \vec{o})}}{\sqrt[3]{\kappa\left(M_{t}\right)}}\right)^{2},
\end{array}
$$

where $\kappa\left(M_{r}\right)$ and $\kappa\left(M_{t}\right)$ are normalization constants for each measurement set such that the weighted reflection error is similar to the weighted transmission error. We define the hemispherical diffuse reflectance $\kappa\left(M_{t}\right)$ and transmission $\kappa\left(M_{t}\right)$ as

$$
\begin{aligned}
& \kappa(M)= \\
& =\frac{2 \pi^{2}}{N_{i}} \sum_{\theta_{i}=0}^{\pi / 2}\left(\frac{\cos \theta_{i} \sin \theta_{i}}{N_{o}} \sum_{\theta_{o}=0}^{\pi / 2} \sum_{\phi_{o}=0}^{\pi} M(\vec{i}, \vec{o}) \cos \theta_{o} \sin \theta_{o}\right),
\end{aligned}
$$

where $N_{i}$ and $N_{o}$ are the number of sampled $\vec{i}$ and $\vec{o}$ angles, 
M. Papas, K. de Mesa, \& H. W. Jensen / A Physically-Based BSDF for Modeling the Appearance of Paper

\begin{tabular}{rccccccccccc}
\hline Paper Type & $\sigma_{s}$ & $\sigma_{a}$ & $g_{b}$ & $w_{b}$ & $g_{f}$ & $w_{f}$ & $d$ & $\eta_{f}$ & $m_{f}$ & $\eta_{b}$ & $m_{b}$ \\
\hline Matte Paper & 81.380 & 0.001 & -0.841 & 0.003 & 0.335 & 0.997 & 0.262 & 1.290 & $0.419_{G G X}$ & 1.550 & $0.892_{G G X}$ \\
Luster Photo Paper & 113.434 & 0.069 & -0.798 & 0.012 & 0.667 & 0.988 & 0.254 & 1.205 & $0.046_{\text {Beck }}$ & 1.766 & $0.9633_{G G X}$ \\
Glossy Photo Paper & 199.789 & 0.060 & -0.673 & 0.042 & 0.842 & 0.958 & 0.254 & 1.161 & $0.037_{\text {Beck }}$ & 1.660 & $0.910_{G G X}$ \\
\hline
\end{tabular}

Table 2: $\vec{P}_{\text {opt }}$ for the Paper BSDF.
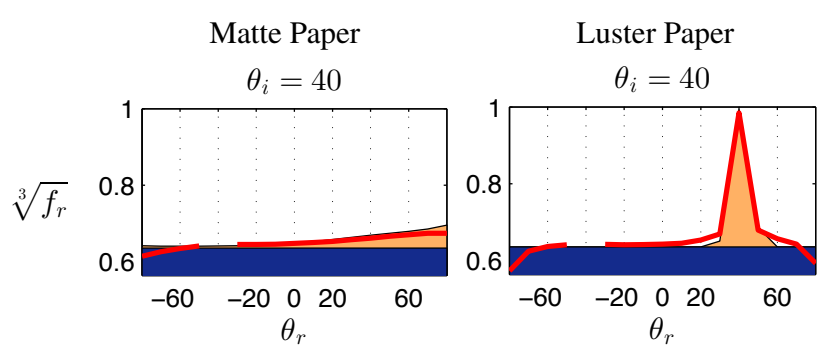

(a) Lambertian with Rough Glass BRDF fits

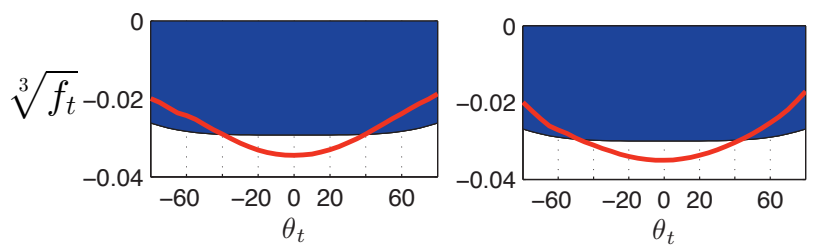

(b) reduced multi-layer BSDF fits
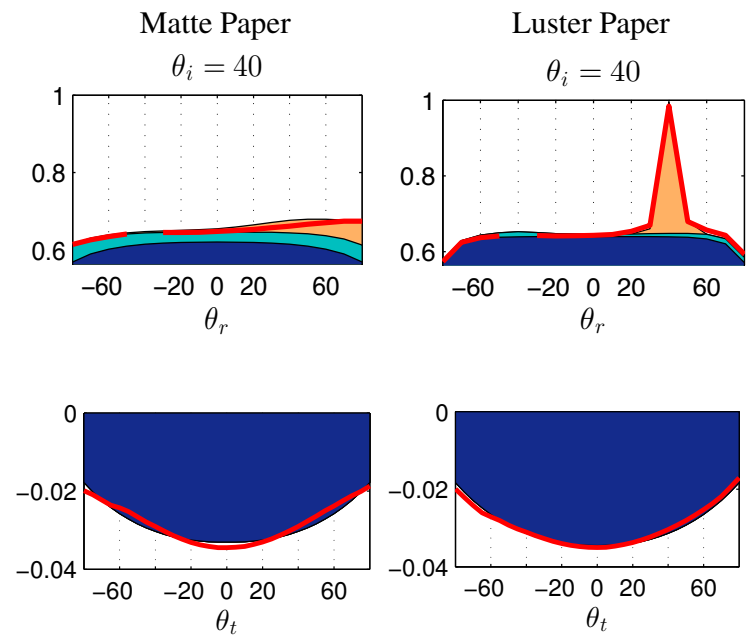

(c) Paper BSDF fits

Figure 9: Fitting quality comparisons between (a) Lambertian with Rough Glass BRDF, (b) reduced multi-layer BSDF, and (c) our Paper BSDF. Red lines indicate the actual BSDF measurements for matte (first and third columns) and luster (second and fourth columns) paper. Surface reflection and single scattering are labeled in orange and cyan, respectively. Multiple scattering regions are marked in blue, with the exception of the Lambertian plots, which mark the diffuse term in the same color.

respectively. For more information on $\vec{P}_{\text {valid }}$ values, consult our supplemental materials.

Since we are fitting both reflection and transmission simultaneously, we use normalization constants $\kappa$ to account for the reflection values being orders of magnitude greater than the transmission values. Otherwise, important information given by transmission measurements will be ignored and the optimization algorithm will overfit the reflection measurements. Additionally, our error function (Equation 19) uses the mean square error (MSE) of the cube roots between the measurements and BSDF values for the given set of parameters. We compute our error function over the the cube root of the measurements due to the extremely high peaks observed both at grazing angles for matte paper, as well as near-reflection directions for luster/glossy paper (Figure 5). These peaks tend to have values that are orders of magnitude greater than the majority of the measurements, which ultimately lead to over-fitting these areas and ignoring the majority of the measurements. Applying a cube root to our measurements before evaluating the mean square error effectively assigns less weight to these peaks in our fitting algorithm.

For fitting we use the SQP [GMW81] minimization al- gorithm implementation found in Matlab. The SQP algorithm works to minimize a scalar-valued nonlinear function of $\left|\vec{P}_{\text {opt }}\right|=N$ real variables using only function values and without any analytic gradient information. For initial values of the optimized parameters, we chose a uniformly random set from $\vec{P}_{\text {opt }}$. Then, we run the optimization up to 5 times, and choose the solution with the least amount of error.

We found that there is parameter crosstalk in our Paper BSDF. By increasing the amount of backscattering in the phase function and the scattering albedo, we observe an increase on the amount of reflected light and vice versa. This incurred many local minima when we attempted to fit the reflectance (BRDF) measurements only. We also observed a dramatic decrease of local minima when we performed fitting on all of the BSDF measurements simultaneously. Minor crosstalk still exists within the multi-lobed phase function model. More specifically, some combinations of mean cosine values and weights of the lobes can provide similar results for multiple scattering and single scattering. We believe that better physically-based analytic phase function models can be used alleviate these issues. 


\section{Results and Comparisons}

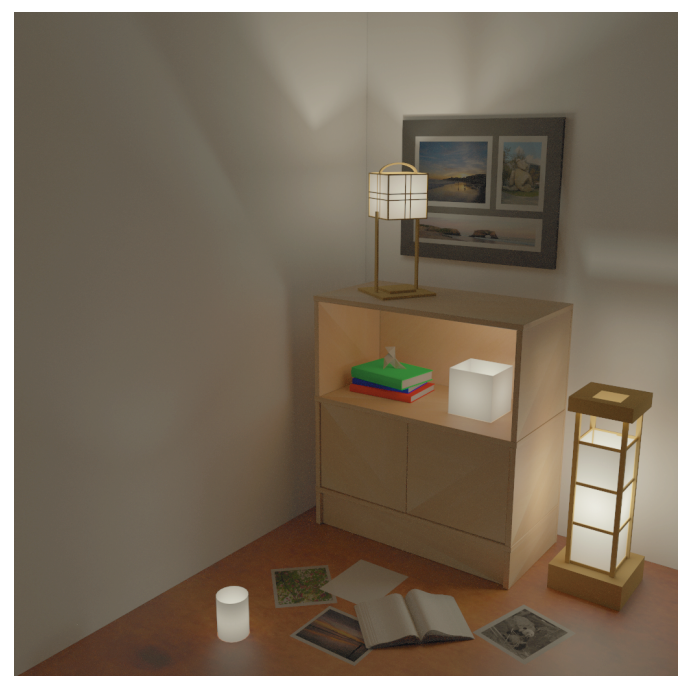

Figure 10: A room scene showcasing the Paper BSDF.

Using our fitting algorithm, we generated a set of optimal parameters for matte, luster, and glossy paper (see Table 2). We implemented our BSDF in pbrt version 2 [PH10].

Figures 1 and 10 showcase the Paper BSDF in two different scenes. In Figure 1, the middle image consists of the full Paper BSDF with matte parameters, while the left column consists of the Paper BSDF rendered at $0.05 \mathrm{~mm}$ thickness. These images offer insight on how paper thickness affects the overall appearance. The rightmost image in Figure 1 is a comparison rendering between Paper BSDF (matte) and a photograph, showing a good match. The room rendering in Figure 10 contains a room with paper lamps and demonstrates the light attenuation that occurs with our BTDF component.

Figure 9 offers detailed plots for the Paper BSDF, a combination of Lambertian and Rough Glass BRDFs, and the reduced multi-layer BSDF fitted against our BSDF measurements. In the reflection case (top), we observe that the Rough Glass BRDF and a Lambertian component cannot adequately capture the decrease in reflectance at grazing reflection angles. This decrease is due to attenuation of multiple scattered light from the surface. In the transmission case (bottom), we show that the previous formulation of light attenuation through rough surfaces introduced by [DWd*08] does not accurately capture the angular distribution of transmitted light. Our results show that the Paper BSDF achieves close fits against our BSDF measurements. In supplemental, we provide more detailed plots of the Lambertian/Rough Glass BRDFs and the multi-layer BSDF fitted against our measurements.

\section{Conclusion and Future Work}

We have presented a BSDF for paper. From our BSDF measurements, we find that paper exhibits specular reflection, retroflection, surface sheen at grazing angles, and subsurface scattering. The surface related features can also differ on each side of paper. We introduce the first method to efficiently measure a homogeneous BSSRDF without any loss of information by converting it into a BSDF. To account for light attenuation through rough surfaces, we present our attenuation term that closely matches our measurements.

We believe this work can be extended to other types of thin, rough materials that exhibit subsurface scattering, such as cloth, clay, porcelain, thin wood, and other artistic media (like canvas). Since paper is both highly scattering and optically thick, we found that the multi-layer BSSRDF [DJ05] is a good choice for modeling paper's appearance. For darker and less optically thick materials, more recent models can be used, such as Quantized-Diffusion [DI11] or Photon Beam Diffusion [HCJ13]. In the future, we would like to fully investigate our model's capabilities in accurately simulating other similar materials, as well as study paper's fluorescent properties using spectral measurements.

We would also like to verify that our recovered model parameters (Table 2) are actually physically accurate. This can be done similarly to the method presented in Narasimhan et al. [NGD*06]. Given this parameter verification, the actual physical parameters produced by our method can then be used by any appearance model, such as the modified multi-layer BSSRDF or even a full Monte Carlo simulation.

Previous research related to accurate soft proofing also encompasses our work. Soft proofing, a representation of a final print on a display device, requires an accurate simulation of ink colors and paper to be effective. While previous work relies on accurate image, display, and printer profiles, our work follows Patil et al. [PFJ04], who use the Phong-Blinn model to accurately render gloss properties seen in actual hard copy prints. Gatt et al. [GWB06] provide a BRDF dataset of commonly used inks and papers for a more complete rendering of printer paper, as well as employ the Cook-Torrance [CT82] model for surface reflections. We believe that our model that be used to improve current soft proofing techniques.

We believe that the acquisition time of appearance measurements can be greatly reduced by utilizing specialized physically-based models for a specific class of materials. This can potentially allow for focusing measurements on regions of the domain where known characteristic appearance features exist. With the feature measurements, one can generate a fit of a specialized physically-based model and perform an informed extrapolation for the remainder of the domain.

Acknowledgments. We would like to thank Neel Joshi, Toshiya Hachisuka, Iman Sadeghi, Wojciech Jarosz, Wenzel Jakob, and Joachim De Deken for their help and feedback during our project. Support for this research was provided, in part, by the California Institute for Telecommunications and Information Technology (Calit2) and the National Science Foundation grants NSF EIA-0303622 and NSF EIA-0224431. 


\section{References}

[BCS64] Bartman F. L., Chaney L. W., Surh M.: Reflectance of Kodak White Paper. Tech. rep., University of Michigan, 1964. 2

[Bli77] BLINN J. F.: Models of light reflection for computer synthesized pictures. SIGGRAPH Comput. Graph. 11, 2 (July 1977), 192-198. 2

[Che09] CHEN T.: Bulk Scattering Approximations for Collimated Light Transmitted through Paper. Master's thesis, University of Waterloo, 2009. 2

[CT82] COOK R. L., TORRANCE K. E.: A reflectance model for computer graphics. ACM Trans. Graph. 1, 1 (Jan. 1982), 7-24. 2, 9

[DI11] D'EON E., IRVING G.: A quantized-diffusion model for rendering translucent materials. ACM Trans. Graph. 30, 4 (July 2011), 56:1-56:14. 2, 6, 9

[DJ05] DONNER C., JENSEN H. W.: Light diffusion in multilayered translucent materials. ACM Trans. Graph. 24, 3 (July 2005), 1032-1039. 2, 3, 6, 7, 9

[dL07] D'EOn E., LuEBKE D.: GPU Gems 3 - Advanced Techniques for Realistic Real-Time Skin Rendering. Addison-Wesley, 2007, ch. 14, pp. 293-345. 6

[DWd*08] DONNER C., WEYRICH T., D'EON E., RAMAMOORTHI R., RUSINKIEWICZ S.: A layered, heterogeneous reflectance model for acquiring and rendering human skin. ACM Transactions on Graphics 27, 5 (2008), 1-12.9

[DWL*09] DAI Q., WANG J., LiU Y., SNyder J., Wu E., GuO B.: The dual-microfacet model for capturing thin transparent slabs. Proc. of Pacific Graphics 28, 7 (2009), 1917-1925. 2

[FSM06] Fabritius T., SaARela J., Myllyla R.: Determination of the refractive index of paper with clearing agents. Proc. of SPIE 6053 (2006), 60530X-60530X-8. 2

[GHP*08] Ghosh A., Hawkins T., PeErs P., Frederiksen S., DEBEVEC P.: Practical modeling and acquisition of layered facial reflectance. ACM Trans. Graph. 27, 5 (Dec. 2008), 139:1139:10. 3, 6

[GLL00] Green K., LAmberg L., Lumme K.: Stochastic modeling of paper structure and monte carlo simulation of light scattering. Proc. of Applied Optics 39, 25 (Sep 2000). 2

[GLL*04] Goesele M., Lensch H. P. A., LANG J., Fuchs C., SEIDEL H.-P.: Disco: Acquisition of translucent objects. ACM Transactions on Graphics 23, 3 (2004), 835-844. 3

[GMW81] Gill P. E., Murray W., Wright M. H.: Practical Optimization. Academic Press, London, 1981. 8

[GWB06] Gatt A., Westland S., BAla R.: Increasing the dimensionality of soft proofing: Gloss and material. In Proc. of Color and Imaging Conference (CIC '06) (2006). 9

[HCJ13] Habel R., Christensen P. H., Jarosz W.: Photon beam diffusion: A hybrid monte carlo method for subsurface scattering. In Computer Graphics Forum (Proceedings of EGSR '13) (June 2013), vol. 32. 2, 6, 9

[HK93] HANRAHAN P., KRUEGER W.: Reflection from layered surfaces due to subsurface scattering. In Proceedings of the 20th Annual Conference on Computer Graphics and Interactive Techniques (New York, NY, USA, 1993), SIGGRAPH '93, ACM, pp. 165-174. 2, 3, 6, 7

[JMLH01] JENSEN H. W., MARSChNER S. R., LeVoy M., HANRAHAN P.: A practical model for subsurface light transport. In Proceedings of the 28th Annual Conference on Computer Graphics and Interactive Techniques (New York, NY, USA, 2001), SIGGRAPH '01, ACM, pp. 511-518. 3
[Mah95] Mahan G. D.: Diffusion Through a Slab. Journal of Mathematical Physics 36 (Dec. 1995), 6758-6773. 3

[MPBM03] MatusiK W., Pfister H., Brand M., McMillan L.: Efficient isotropic brdf measurement. In Computer Graphics Forum (Proc. of EGSR '03) (2003), pp. 241-247. 3

[NGD*06] Narasimhan S. G., Gupta M., Donner C., RAMAMOORTHI R., NAYAR S. K., JENSEN H. W.: Acquiring scattering properties of participating media by dilution. $A C M$ Transactions on Graphics 25, 3 (2006), 1003-1012. 9

[ON94] OREN M., NAYAR S. K.: Generalization of lambert's reflectance model. In Proceedings of the 21st Annual Conference on Computer Graphics and Interactive Techniques (New York, NY, USA, 1994), SIGGRAPH '94, ACM, pp. 239-246. 2

[PCW89] Patterson M. S., Chance B., Wilson B. C.: Time resolved reflectance and transmittance for the noninvasive measurement of tissue optical properties. Proc. of Applied Optics 28, 12 (Jun 1989), 2331-2336. 3

[PFJ04] PATIL R. A., FAIRChild M. D., Johnson G. M.: 3d simulation of prints for improved soft-proofing. In Proc. of Color and Imaging Conference (CIC '04) (2004), pp. 193-199. 9

[PH10] PhARR M., HuMPhreYs G.: Physically based rendering. From theory to implementation. Morgan Kaufmann, 2010. 9

[PvBM*06] PeErs P., Vom Berge K., Matusik W., RaMAmoorthi R., LAWrence J., Rusinkiewicz S., Dutré P.: A compact factored representation of heterogeneous subsurface scattering. ACM Trans. Graph. 25, 3 (July 2006), 746-753. 3

[Sta01] StAm J.: An illumination model for a skin layer bounded by rough surfaces. In Proc. of EGWR '01 (London, UK, UK, 2001), Springer-Verlag, pp. 39-52. 2

[TS67] TORRANCE K. E., Sparrow E. M.: Theory for offspecular reflection from roughened surfaces. J. Opt. Soc. Amer, 57 (1967), 1105-1114. 2

[TWL*05] Tong X., WANG J., Lin S., Guo B., SHuM H.-Y.: Modeling and rendering of quasi-homogeneous materials. ACM Transactions on Graphics 24, 3 (2005), 1054-1061. 3

[vGSK98] VAN GinneKEn B., StAVRIDi M., KoENDERINK J. J.: Diffuse and specular reflectance from rough surfaces. Proc. of Applied Optics 37, 1 (1998), 130-139. 2

[War92] WARD G. J.: Measuring and modeling anisotropic reflection. SIGGRAPH Comput. Graph. 26, 2 (July 1992), 265-272. 2

[WMLT07] Walter B., Marschner S. R., Li H., TorRance K. E.: Microfacet models for refraction through rough surfaces. In Computer Graphics Forum (Proceedings of EGSR '07) (June 2007), pp. 195-206. 2, 3, 5, 6

[WW07] WeIdLICH A., WilkiE A.: Arbitrarily layered microfacet surfaces. In Proc. of GRAPHITE '07 (Dec. 2007), ACM, pp. 171-178. 2

[WZT*08] WANG J., ZhaO S., TONG X., Lin S., Lin Z., Dong Y., Guo B., SHum H.-Y.: Modeling and rendering of heterogeneous translucent materials using the diffusion equation. ACM Transactions on Graphics 27, 1 (2008), 1-18. 3 